\title{
A subexponential upper bound for van der Waerden numbers $W(3, k)$
}

\author{
Tomasz Schoen \\ Faculty of Mathematics and Computer Science \\ Adam Mickiewicz University \\ Uniwersytetu Poznańskiego 4 \\ 61-614 Poznań, Poland \\ schoen@amu.edu.pl
}

Submitted: Jul 9, 2020; Accepted: May 23, 2021; Published: Jun 4, 2021

(C) The author. Released under the CC BY license (International 4.0).

\begin{abstract}
We show an improved upper estimate for van der Waerden number $W(3, k)$ : there is an absolute constant $c>0$ such that if $\{1, \ldots, N\}=X \cup Y$ is a partition such that $X$ does not contain any arithmetic progression of length 3 and $Y$ does not contain any arithmetic progression of length $k$ then

$$
N \leqslant \exp \left(O\left(k^{1-c}\right)\right) .
$$
\end{abstract}

Mathematics Subject Classifications: 05D10, 11B25

\section{Introduction}

Let $k$ and $l$ be positive integers. The van der Waerden number $W(k, l)$ is the smallest positive integer $N$ such that in any partition $\{1, \ldots, N\}=X \cup Y$ there is an arithmetic progression of length $k$ in $X$ or an arithmetic progression of length $l$ in $Y$. The existence of such numbers was established by van der Waerden [22], however the order of magnitude of $W(k, l)$ is unknown for $k, l \geqslant 3$. Clearly, $W(k, l)$ is related to Szemerédi's theorem on arithmetic progressions [20] and any effective estimate in this theorem leads to an upper bound on the van der Waerden numbers. Currently the best known bounds in the most important diagonal case are

$$
(1-o(1)) \frac{2^{k-1}}{e k} \leqslant W(k, k) \leqslant 2^{2^{2^{2^{k+9}}}} .
$$

The upper bound follows from the famous work of Gowers [12] and the lower bound was proved by Szabó [19] using a probabilistic argument. Furthermore, Berlekamp [3] showed 
that if $k-1$ is a prime number then

$$
W(k, k) \geqslant(k-1) 2^{k-1}
$$

Another very intriguing instance of the problem is the estimation of the numbers $W(3, k)$, as these are related to Roth's theorem [15] concerning estimates for sets avoiding three-term arithmetic progressions. Let us denote by $r(N)$ the size of the largest progression-free subset of $\{1, \ldots, N\}$. We know that

$$
r(N) \ll \frac{N}{\log N^{1-o(1)}},
$$

see $[4,5,17,18]$, which implies $W(3, k) \leqslant \exp \left(O\left(k^{1+o(1)}\right)\right)$.

Green [13] proposed a very clever argument based on arithmetic properties of sumsets to bound $W(3, k)$. Building on this method and applying results from [10] it was shown in [11] that

$$
W(3, k) \leqslant \exp (O(k \log k)) .
$$

The best known lower bound was obtained by Li and Shu [14] (see also [8]), who showed that

$$
W(3, k) \gg\left(\frac{k}{\log k}\right)^{2}
$$

The purpose of this paper is to prove a subexponential bound on $W(3, k)$.

Theorem 1. There are absolute constants $C, c>0$ such that for every $k$ we have

$$
W(3, k) \leqslant \exp \left(C k^{1-c}\right) .
$$

Our argument is based on the method of [18], which explores in details the structure of a large spectrum. This method can be partly applied (see Lemma 5) in our approach and it deals only with a progression-free partition class. The second part of the proof exploits the structure of both partition classes and in this case the argument of [18] has to be significantly modified.

Let us remark that during the review process a preprint of Bloom and Sisask [6], which improves an upper bound in Roth's theorem to $N /(\log N)^{1+c}$ for $c \approx 2^{-2^{2^{1000}}}$, has appeared. That result implies directly that $W(3, k) \leqslant \exp \left(C k^{1-c}\right)$ with $c \approx 2^{-2^{2^{1000}}}$.

\section{Notation}

Given functions $f, g: \mathbb{Z} / N \mathbb{Z} \rightarrow \mathbb{C}$, the convolution of $f$ and $g$ is defined by

$$
(f * g)(x)=\sum_{t \in \mathbb{Z} / N \mathbb{Z}} f(t) g(x-t) .
$$


The Fourier coefficients of a function $f: \mathbb{Z} / N \mathbb{Z} \rightarrow \mathbb{C}$ are defined by

$$
\widehat{f}(r)=\sum_{x=0}^{N-1} f(x) e^{-2 \pi i x r / N},
$$

where $r \in \mathbb{Z} / N \mathbb{Z}$. The inversion formula states that

$$
f(x)=\frac{1}{N} \sum_{x=0}^{N-1} \widehat{f}(r) e^{2 \pi i x r / N} .
$$

We denote by $1_{A}(x)$ the indicator function of set $A$. Thus using the inversion formula and the fact that $\widehat{(f * g)}(r)=\widehat{f}(r) \widehat{g}(r)$ one can express the number of three-term arithmetic progressions (including trivial ones) in a set $A \subseteq \mathbb{Z} / N \mathbb{Z}$ by

$$
\frac{1}{N} \sum_{r=0}^{N-1} \widehat{1_{A}}(r)^{2} \widehat{1_{A}}(-2 r) \text {. }
$$

Parseval's identity asserts in particular that

$$
\sum_{r=0}^{N-1}\left|\widehat{1_{A}}(r)\right|^{2}=|A| N .
$$

Let $\theta \geqslant 0$ be a real number. The $\theta$-spectrum of $A$ is defined by

$$
\Delta_{\theta}(A)=\left\{r \in \mathbb{Z} / N \mathbb{Z}:\left|\widehat{1_{A}}(r)\right| \geqslant \theta|A|\right\} .
$$

If $A$ is specified then we write $\Delta_{\theta}$ instead of $\Delta_{\theta}(A)$.

By the span of a finite set $S$ we mean

$$
\operatorname{Span}(S)=\left\{\sum_{s \in S} \varepsilon_{s} s: \varepsilon_{s} \in\{-1,0,1\} \text { for all } s \in S\right\}
$$

and the dimension of $A$ is defined by

$$
\operatorname{dim}(A)=\min \{|S|: A \subseteq \operatorname{Span}(S)\} .
$$

Chang's Spectral Lemma provides an upper bound for the dimension of a spectrum.

Lemma 2. [9] Let $A \subseteq \mathbb{Z} / N \mathbb{Z}$ be a set of size $|A|=\delta N$ and let $\theta>0$. Then

$$
\operatorname{dim}\left(\Delta_{\theta}(A)\right) \ll \theta^{-2} \log (1 / \delta) .
$$

We are going to use Bohr sets [7] to prove the main result. Let $\Gamma \subseteq \widehat{G}$ and $\gamma \in\left(0, \frac{1}{2}\right]$ then the Bohr set generated by $\Gamma$ with radius $\gamma$ is

$$
B(\Gamma, \gamma)=\{x \in \mathbb{Z} / N \mathbb{Z}:\|t x / N\| \leqslant \gamma \text { for all } t \in \Gamma\},
$$

where $\|x\|=\min _{y \in \mathbb{Z}}|x-y|$. The rank of $B$ is the size of $\Gamma$ and we denote it by $\operatorname{rk}(B)$. Given $\eta>0$ and a Bohr set $B=B(\Gamma, \gamma)$, by $B_{\eta}$ we mean the Bohr set $B(\Gamma, \eta \gamma)$. We will also use the notation $\beta=\frac{1}{|B|} 1_{B}$. We will use two basic properties of Bohr sets concerning their size and regularity, see [21]. 
Lemma 3. [7] For every $\gamma \in\left(0, \frac{1}{2}\right]$ we have

$$
\gamma^{|\Gamma|} N \leqslant|B(\Gamma, \gamma)| \leqslant 8^{|\Gamma|+1}|B(\Gamma, \gamma / 2)|
$$

We call a Bohr set $B(\Gamma, \gamma)$ regular if for every $\eta$, where $|\eta| \leqslant 1 /(100|\Gamma|)$ we have

$$
(1-100|\Gamma||\eta|)|B| \leqslant\left|B_{1+\eta}\right| \leqslant(1+100|\Gamma||\eta|)|B| .
$$

Bourgain [7] showed that regular Bohr sets are ubiquitous.

Lemma 4. [7] For every Bohr set $B(\Gamma, \gamma)$, there exists $\gamma^{\prime}$ such that $\frac{1}{2} \gamma \leqslant \gamma^{\prime} \leqslant \gamma$ and $B\left(\Gamma, \gamma^{\prime}\right)$ is regular.

\section{Proof of Theorem 1}

Our main tool is the next lemma, which can be extracted from [18], by conjugation of results concerning the case of 'small' Fourier coefficients (see Lemmas 7 and 9 of [18]) and the case of 'middle' Fourier coefficients (Lemmas 12 and 13 of [18]). Its proof makes use of the deep result by Bateman and Katz in $[1,2]$ describing the structure of the large spectrum. The case of 'large' Fourier coefficients is treated similarly as in [18], however using partition properties we will be able to obtain much better estimate.

Lemma 5. [18] There exists an absolute constant $c>0$ such that the following holds. Let $A \subseteq \mathbb{Z} / N \mathbb{Z},|A|=\delta N$ be a set such that

$$
\sum_{r: \delta^{1+c}|A| \leqslant\left|\widehat{1_{A}}(r)\right| \leqslant \delta^{1 / 10}|A|}\left|\widehat{1_{A}}(r)\right|^{3} \geqslant \frac{1}{10} \delta^{c / 5}|A|^{3} .
$$

Then there is a regular Bohr set $B$ with $\operatorname{rk}(B) \ll \delta^{-1+c}$ and radius $\Omega\left(\delta^{1-c}\right)$ such that for some $t$

$$
|(A+t) \cap B| \gg \delta^{1-c}|B|
$$

Furthermore, we apply Bloom's iterative lemma, that provides a density increment by a constant factor greater than 1 for progression-free sets and Sanders' lemma on a containment of long arithmetic progressions in dense subsets of regular Bohr sets.

Lemma 6. [4] There exists an absolute constant $c_{1}>0$ such that the following holds. Let $B \subseteq \mathbb{Z} / N \mathbb{Z}$ be a regular Bohr set of rank d. Let $A_{1} \subseteq B$ and $A_{2} \subseteq B_{\varepsilon}$, each with relative densities $\alpha_{i}$. Let $\alpha=\min \left(c_{1}, \alpha_{1}, \alpha_{2}\right)$ and assume that $d \leqslant \exp \left(c_{1}\left(\log ^{2}(1 / \alpha)\right)\right.$. Suppose that $B_{\varepsilon}$ is also regular and $c_{1} \alpha /(4 d) \leqslant \varepsilon \leqslant c_{1} \alpha / d$. Then either

(i) there is a regular Bohr set $B^{\prime}$ of rank $\operatorname{rk}\left(B^{\prime}\right) \leqslant d+O\left(\alpha^{-1} \log (1 / \alpha)\right)$ and size

$$
\left|B^{\prime}\right| \geqslant \exp \left(-O\left(\log ^{2}(1 / \alpha)\left(d+\alpha^{-1} \log (1 / \alpha)\right)\right)\right)|B|
$$

such that

$$
\left|\left(A_{1}+t\right) \cap B^{\prime}\right| \gg\left(1+c_{1}\right) \alpha_{1}\left|B^{\prime}\right|
$$

for some $t \in \mathbb{Z} / N \mathbb{Z}$; 
(ii) or there are $\Omega\left(\alpha_{1}^{2} \alpha_{2}\left|B \| B_{\varepsilon}\right|\right)$ three-term arithmetic progressions $x+y=2 z$ with $x, y \in A_{1}, z \in A_{2}$;

Lemma 7. [16] Let $B(\Gamma, \gamma) \subseteq \mathbb{Z} / N \mathbb{Z}$ be a regular Bohr set of rank $d$ and let $\varepsilon$ be a positive number satisfying $\varepsilon^{-1} \ll \gamma d^{-1} N^{1 / d}$. Suppose that $A \subseteq B$ contains at least a proportion $1-\varepsilon$ of $B(\Gamma, \gamma)$. Then $A$ contains an arithmetic progression of length at least $1 /(4 \varepsilon)$.

Furthermore, to apply Bloom's result we will need to prove a standard fact on Bohr sets. Let us also remark that it follows from the proof of Bloom's lemma that we can take $c_{1}<1 / 1000$. We will use the following basic property of Bohr sets.

Lemma 8. [11] Let $B$ be a regular Bohr set of rank $d$ and radius $\gamma$ and let $A$ be a set with $|A|=\alpha|B|$. Suppose that $\varepsilon<\kappa \alpha /(100 d)$ for some $\kappa \in(0,1)$. Then

$$
\sum_{x \in B}\left(1_{A} * 1_{B_{\varepsilon}}\right)(x) \geqslant(1-\kappa) \alpha|B|\left|B_{\varepsilon}\right| .
$$

Lemma 9. Let $B$ be a regular Bohr set of rankd and suppose that $A \subseteq B$ and $|A|=\alpha|B|$. Then there is $\varepsilon$ with $c_{1} \alpha /(4 d) \leqslant \varepsilon \leqslant c_{1} \alpha / d$ ( $c_{1}$ is a constant given by Lemma 6 ), and regular Bohr sets $B^{\prime}$ and $B_{\varepsilon}^{\prime}$ of rank $d$ and size

$$
\left|B^{\prime}\right| \geqslant \exp (-O(d \log (1 / \alpha) \log (d / \alpha)))|B|
$$

such that

$$
\left|(A+t) \cap B^{\prime}\right| \geqslant \frac{1}{2} \alpha\left|B^{\prime}\right| \quad \text { and } \quad\left|(A+t) \cap B_{\varepsilon}^{\prime}\right| \geqslant \frac{1}{2} \alpha\left|B_{\varepsilon}^{\prime}\right|
$$

for some $t$.

Proof. Let $\varepsilon_{1}$ and $\varepsilon_{2}$ be any numbers satisfying $c_{1} \alpha /(4 d) \leqslant \varepsilon_{1}, \varepsilon_{2} \leqslant c_{1} \alpha / d$ and such that the Bohr sets $B^{1}=B_{\varepsilon_{1}}$ and $B^{2}=B_{\varepsilon_{1} \varepsilon_{2}}$ are regular. Put $\beta_{1}=\frac{1}{\left|B_{1}\right|} 1_{B_{1}}$ and $\beta_{2}=\frac{1}{\left|B_{2}\right|} 1_{B_{2}}$ then by Lemma 8 we have

$$
\sum_{x}\left(\left(\beta_{1} * 1_{A}\right)(x)+\left(\beta_{2} * 1_{A}\right)(x)\right) \geqslant \frac{9}{5} \alpha|B|,
$$

hence for some $x$ we have

$$
\left(\beta_{1} * 1_{A}\right)(x)+\left(\beta_{2} * 1_{A}\right)(x) \geqslant \frac{9}{5} \alpha .
$$

If

$$
\left(\beta_{1} * 1_{A}\right)(x) \geqslant \frac{1}{2} \alpha \quad \text { and } \quad\left(\beta_{2} * 1_{A}\right)(x) \geqslant \frac{1}{2} \alpha
$$

then we can put $B^{\prime}=B^{1}$. Otherwise, for some $i \in\{1,2\}$ we have $\left(\beta_{i} * 1_{A}\right)(x) \geqslant(6 / 5) \alpha$ and we put $B=B^{i}$ and apply the same procedure again. Clearly, we can iterate this procedure $O(\log (1 / \alpha))$ times, as the density of a set can not exceed 1 . Thus after $O(\log (1 / \alpha))$ iterative steps we obtain a pair of Bohr sets $B^{\prime}, B_{\varepsilon}^{\prime}$ satisfying (3). From Lemma 3 it follows that

$$
\left|B^{\prime}\right| \geqslant \exp (-O(d \log (1 / \alpha) \log (d / \alpha)))|B|,
$$

which concludes the proof. 
Proof of Theorem 1. Put $M=W(3, k)-1$ and let $\{1, \ldots, M\}=X \cup Y$ be a partition such that $X$ and $Y$ avoid 3 and $k$-term arithmetic progressions respectively. Clearly, we may assume that $M \geqslant 100 k$ hence

$$
|Y| \leqslant M-\lfloor M / k\rfloor \leqslant M-M /(2 k),
$$

as no block of $k$ consecutive numbers is contained in $Y$ and therefore $|X| \geqslant M /(2 k)$. Let $N$ be any prime number satisfying $2 M<N \leqslant 4 M$. We embed $\{1, \ldots, M\}=X \cup Y$ in $\mathbb{Z} / N \mathbb{Z}$ in a natural way and observe that $\{1, \ldots, M\}$ considered as a subset of $\mathbb{Z} / N \mathbb{Z}$ is 2 Freiman isomorphic to $\{1, \ldots, M\}$ considered as a subset of $\mathbb{Z}$ and therefore any arithmetic progression in $\{1, \ldots, M\} \subseteq \mathbb{Z} / N \mathbb{Z}$ is a genuine progression in $\mathbb{Z}$. Put $|X|=\delta N$ and note that we can assume that $\delta \gg(\log N)^{-1.1}$. First let us assume that

$$
\sum_{r: \delta^{1+c}|X| \leqslant\left|\widehat{1_{X}}(r)\right| \leqslant \delta^{1 / 10}|X|}\left|\widehat{1_{X}}(r)\right|^{3} \geqslant \delta^{c / 5}|X|^{3}
$$

where $c>0$ is the fixed absolute constant from Lemma 5 . Then by Lemma 5 there is $t \in \mathbb{Z} / N \mathbb{Z}$ and a regular Bohr set $B^{0}$ with $\operatorname{rk}\left(B^{0}\right)=d \ll \delta^{-1+c}$ and radius $\Omega\left(\delta^{1-c}\right)$ such that

$$
\left|(X+t) \cap B^{0}\right| \gg \delta^{1-c}|B|
$$

for some absolute constant $c>0$. Writing $X_{0}=(X+t) \cap B_{0}$ we have

$$
\left|X_{0} \cap B^{0}\right| \gg \alpha\left|B^{0}\right|
$$

where

$$
\alpha \gg \delta^{1-c},
$$

and by Lemma 3

$$
\left|B^{0}\right| \geqslant \exp \left(-O\left(\delta^{-1+c} \log (1 / \delta)\right)\right) N .
$$

By Lemma 9 there is $\varepsilon \gg \alpha^{2}$ and regular Bohr sets $B^{\prime}$ and $B_{\varepsilon}^{\prime}$ of rank $d$ such that

$$
\left|(A+t) \cap B^{\prime}\right| \geqslant \frac{1}{2} \alpha\left|B^{\prime}\right| \text { and }\left|(A+t) \cap B_{\varepsilon}^{\prime}\right| \geqslant \frac{1}{2} \alpha\left|B_{\varepsilon}^{\prime}\right|,
$$

and

$$
\left|B^{\prime}\right| \geqslant \exp \left(-O\left(\alpha^{-1} \log ^{2}(1 / \alpha)\right)\right)\left|B^{0}\right| \geqslant \exp \left(-O\left(\delta^{-1+c} \log ^{2}(1 / \delta)\right)\right) N .
$$

Next, we iteratively apply Lemma 6 and Lemma 9 . Since after each step the density increases by factor $1+c_{1}$ it follows that after $l \ll \log (1 / \alpha)$ steps case $(i i)$ of Lemma 6 holds. Let $B^{i}$ be Bohr sets obtained in the iterative procedure. Note that $B^{i}$ has rank $\operatorname{rk}\left(B^{i}\right)=d_{i} \ll \alpha^{-1} \log ^{2}(1 / \alpha)$ for every $i \leqslant l$ and

$$
\left|B^{i+1}\right| \geqslant \exp \left(-O\left(\log ^{2}(1 / \alpha)\left(d_{i}+\alpha^{-1} \log (1 / \alpha)\right)\right)\right)\left|B_{i}\right|
$$

for every $i<l$. Therefore, there are

$$
\Omega\left(\alpha^{3}\left|B^{l}\right|\left|B_{\varepsilon}^{l}\right|\right)
$$


three-term arithmetic progressions in $X$, where $\varepsilon \geqslant c_{1} \alpha /\left(4 \operatorname{rk}\left(B^{l}\right)\right) \gg \alpha^{2} \log ^{2}(1 / \alpha)$. By Lemma 6 and Lemma 3 we have

$$
\left|B^{l}\right| \geqslant \exp \left(-O\left(\alpha^{-1} \log ^{4}(1 / \alpha)\right)\right) N \geqslant \exp \left(-O\left(\delta^{-1+c} \log ^{4}(1 / \delta)\right)\right) N,
$$

and

$$
\begin{aligned}
\left|B_{\varepsilon}^{l}\right| & \geqslant \exp \left(-O\left(\alpha^{-1} \log ^{3}(1 / \alpha)\right)\right) \exp \left(-O\left(\alpha^{-1} \log ^{4}(1 / \alpha)\right)\right) N \\
& \geqslant \exp \left(-O\left(\delta^{-1+c} \log ^{4}(1 / \delta)\right)\right) N .
\end{aligned}
$$

Thus, $X$ contains

$$
\Omega\left(\delta^{3-3 c} \exp \left(-O\left(\delta^{-1+c} \log ^{4}(1 / \delta)\right)\right) N^{2}\right)
$$

arithmetic progressions of length three. Since there are only $|X|$ trivial progressions in $X$ it follows that

$$
|X| \gg \delta^{3-3 c} \exp \left(-O\left(\delta^{-1+c} \log ^{4}(1 / \delta)\right)\right) N^{2},
$$

SO

$$
W(3, k) \ll N \ll \exp \left(O\left(\delta^{-1+c} \log ^{4}(1 / \delta)\right) \leqslant \exp \left(O\left(k^{1-c} \log ^{4} k\right)\right) .\right.
$$

Next let us assume that (4) does not hold. Let us define $\Delta^{\prime}=\Delta_{\delta^{1 / 10}} \cup 2^{-1} \cdot \Delta_{\delta^{1 / 10}}$ and observe that $r \notin \Delta^{\prime}$ is equivalent to $r \notin \Delta_{\delta^{1 / 10}}$ and $2 r \notin \Delta_{\delta^{1 / 10}}$. By Chang's lemma

$$
\operatorname{dim}\left(\Delta^{\prime}\right) \leqslant 2 \operatorname{dim}\left(\Delta_{\delta^{1 / 10}}\right) \ll \delta^{-1 / 5} \log (1 / \delta)
$$

and let $\Lambda$ be any set such that $1 \in \Lambda,|\Lambda| \ll \delta^{-1 / 5} \log (1 / \delta)$ and $\Delta^{\prime} \subseteq \operatorname{Span}(\Lambda)$. Let $B=B(\Lambda, \gamma)$ be a regular Bohr set with radius $\delta^{3} \ll \gamma \leqslant \delta^{3}$. Since $1 \in \Lambda$ it follows that for every $b \in B$ we

$$
\|b / N\| \leqslant \gamma N \leqslant 4 \gamma M .
$$

Recall that $\beta=\frac{1}{|B|} 1_{B}$ then for every $r \in \Delta^{\prime}$ we have

$$
|\widehat{\beta}(r)-1| \leqslant \frac{1}{|B|} \sum_{b \in B}\left|e^{-2 \pi i r b / N}-1\right| \leqslant \frac{2 \pi}{|B|} \sum_{b \in B} \sum_{\lambda \in \Lambda}\|r b / N\| \leqslant 2 \pi \delta^{2},
$$

and similarly $|\widehat{\beta}(2 r)-1| \leqslant 4 \pi \delta^{2}$. For $t \in \mathbb{Z} / N \mathbb{Z}$ put

$$
f(t)=\beta * 1_{X}(t)
$$

and note that if for some $t \in[4 \gamma M,(1-4 \gamma) M]$ we have $f(t)=\frac{1}{|B|}|X \cap(B+t)| \leqslant \delta^{1+c^{\prime}}$, where $c^{\prime}=c / 20$, then since $B+t \subseteq[1, M]$ it follows that

$$
|Y \cap(B+t)| \geqslant\left(1-\delta^{1+c^{\prime}}\right)|B| .
$$

Therefore, by Lemma 7 either $\delta^{-1-c^{\prime}} \gg \gamma d^{-1} N^{1 / d}$ or $Y$ contains an arithmetic progression of length $\frac{1}{4} \delta^{-1-c^{\prime}}$. The former inequality implies that

$$
k^{1+c^{\prime}} \gg \gamma d^{-1} N^{1 / d} \gg \delta^{4} N^{O\left(\delta^{1 / 5} \log ^{-1}(1 / \delta)\right)} \gg k^{-4} N^{O\left(k^{-1 / 5} \log ^{-1} k\right)},
$$


SO

$$
W(3, k) \ll N \ll \exp \left(O\left(k^{1 / 5} \log ^{2} k\right)\right) .
$$

If the second alternative holds then

$$
\frac{1}{4} \delta^{-1-c^{\prime}}<k
$$

hence by (1)

$$
k^{-1 /\left(1+c^{\prime}\right)} \ll \delta \ll(\log N)^{-1+o(1)}
$$

so

$$
W(3, k) \leqslant N \ll \exp \left(O\left(k^{\frac{1}{1+c^{\prime}}+o(1)}\right)\right)
$$

Finally we can assume that for every $t \in[4 \gamma M,(1-4 \gamma) M]$ we have $f(t) \geqslant \delta^{1+c^{\prime}}$. Let $T(X)$ denote the number of three-term arithmetic progressions in $X$ and let

$$
T(f)=\sum_{x+y=2 z} f(x) f(y) f(z) .
$$

Then clearly

$$
T(f) \gg \delta^{3+3 c^{\prime}} M^{2} \gg \delta^{3+c / 6} N^{2}
$$

and we will show that $T(X)$ does not differ much from $T(f)$

$$
\begin{aligned}
|T(X)-T(f)| & =\frac{1}{N}\left|\sum_{r=0}^{N-1} \widehat{1_{X}}(r)^{2} \widehat{1_{X}}(-2 r)-\sum_{r=0}^{N-1} \widehat{f}(r)^{2} \widehat{f}(-2 r)\right| \\
& \leqslant \frac{1}{N} \sum_{r=0}^{N-1}\left|\widehat{1_{X}}(r)^{2} \widehat{1_{X}}(-2 r)\left(1-\widehat{\beta}(r)^{2} \widehat{\beta}(-2 r)\right)\right| \\
& =S_{1}+S_{2},
\end{aligned}
$$

where $S_{1}$ and $S_{2}$ are summations of (7) respectively over $\Delta^{\prime}$ and $\mathbb{Z} / N \mathbb{Z} \backslash \Delta^{\prime}$. By (5), the negation of (4), Parseval's formula and Hölder's inequality we have

$$
\begin{aligned}
S_{1} & \ll \delta^{2} \frac{1}{N} \sum_{r \in \Delta^{\prime}}\left|\widehat{1_{X}}(r)\right|^{3} \leqslant \delta^{3} \sum_{r=0}^{N-1}\left|\widehat{1_{X}}(r)\right|^{2}=\delta^{2}|X|^{2}, \\
S_{2} & \leqslant \frac{2}{N} \sum_{r \notin \Delta^{\prime}}\left|\widehat{1_{X}}(r)^{2} \widehat{1_{X}}(-2 r)\right| \\
& \leqslant \frac{2}{N}\left(\sum_{r \notin \Delta^{\prime}}\left|\widehat{1_{X}}(r)\right|^{3}\right)^{2 / 3}\left(\sum_{r \notin \Delta^{\prime}}\left|\widehat{1_{X}}(2 r)\right|^{3}\right)^{1 / 3} \\
& \leqslant \frac{2}{N} \sum_{r \notin \Delta_{\delta^{1} / 10}}\left|\widehat{1_{X}}(r)\right|^{3} \\
& \leqslant \frac{2}{N} \sum_{r \in \Delta_{\delta^{1+c} \backslash \Delta_{\delta^{1 / 10}}}}\left|\widehat{1_{X}}(r)\right|^{3}+\frac{2}{N} \sum_{r \notin \Delta_{\delta^{1}+c}}\left|\widehat{1_{X}}(r)\right|^{3} \\
& \ll \delta^{2}|X|^{2}+\delta^{1+c / 5}|X|^{2}+\delta^{1+c}|X|^{2} \ll \delta^{1+c / 5}|X|^{2}
\end{aligned}
$$


Thus,

$$
|T(X)-T(f)| \ll \delta^{3+c / 5} N^{2},
$$

so by (6) and the fact that $X$ avoids non-trivial three-term arithmetic progression we have

$$
|X|=T(X) \gg \delta^{3+c / 6} N^{2},
$$

hence

$$
W(3, k) \leqslant N \ll \delta^{-2-c / 6} \ll k^{3}
$$

which concludes the proof.

\section{References}

[1] M. Bateman, N. Katz, New bounds on cap sets, Journal of AMS 2 (2012), 585613.

[2] M. Bateman, N. KATZ, Structure in additively nonsmoothing sets, arXiv: 1104.2862v1.

[3] E. BerleKAmp, A construction for partitions which avoid long arithmetic progressions, Canad. Math. Bull. 11 (1968) 409-414.

[4] T. Bloom, A quantitative improvement for Roth's theorem on arithmetic progressions, J. Lond. Math. Soc. 93 (2016), 643-663.

[5] T. Bloom, O. Sisask, Logarithmic bounds for Roth's theorem via almostperiodicity, Discrete Analysis 4 (2019), 20 pp.

[6] T. Bloom, O. Sisask, Breaking the logarithmic barrier in Roth's theorem on arithmetic progressions, arXiv:2007.03528.

[7] J. Bourgain, On triples in arithmetic progression, Geom. Funct. Anal. 9 (1999), 968-984.

[8] T. Brown, B. Landman, A. Robertson, Bounds on some van der Waerden numbers, J. Combin. Theory Ser. A 115 (2008), 1304-1309.

[9] M.-C. Chang, A polynomial bound in Freiman's theorem, Duke Math. J. 3 (2002), 399-419.

[10] E. Croot, I. Ruzsa, T. Schoen, Arithmetic progressions in sparse sumsets, Combinatorial number theory, de Gruyter, Berlin, 2007, 157-164.

[11] K. Cwalina, T. Schoen, Tight bounds on additive Ramsey-type numbers, J. Lond. Math. Soc. 96 (2017), 601-620.

[12] W. T. Gowers, A new proof of Szemerédi's theorem, Geom. Funct. Anal. 11 (2001), 465-588.

[13] B. Green, Arithmetic progressions in sumsets, Geom. Funct. Anal. 12 (2002), 584597.

[14] Y. Li, J. SHU, A lower bound for off-diagonal van der Waerden numbers, Adv. in Appl. Math. 44 (2010), 243-247. 
[15] K. F. Roth, On certain sets of integers, J. London Math. Soc. 28 (1953), 104-109.

[16] T. Sanders, Additive structures in sumsets, Math. Proc. Cambridge Philos. Soc. 144 (2008), 289-316.

[17] T. Sanders, On Roth's Theorem on Progressions, Ann. of Math. 174 (2011), 619636.

[18] T. Schoen, Improved bound in Roth's theorem on arithmetic progressions, Adv. Math. (2021), https://doi.org/10.1016/j.aim.2021.107801 (arXiv:2005.01145).

[19] Z. SzABó, An application of Lovász' local lemma-A new lower bound for the van der Waerden numbers, Random Structures Algorithms 1 (1990), 343-360.

[20] E. SzEMERÉDI, On sets of integers containing no $k$ elements in arithmetic progression, Acta Arith. 27 (1975), 199-245.

[21] T. TAO, V. Vu, Additive combinatorics, Cambridge University Press 2006.

[22] B. L. VAN DER WAERden, Beweis einer Baudetschen Vermutung, Nieuw Arch. Wiskd. 15 (1927), 257-271. 